of clinical symptoms, which may include headache, mental confusion, altered mental status, fatigue, anorexia, cyanosis, shortness of breath, palpitation, tinnitus, dizziness, sleep disturbances, and/or dilatation of veins. These various symptoms and polycythemia generally will resolve at lower altitudes.

We report a case of CMS seen in Andahuaylillas, a small village outside of Cusco $(3360 \mathrm{~m})$, in the Peruvian Andes. A 55 -year-old male farmer presented to our medical clinic during a 2-week project with a local nongovernmental organization (NGO). The patient's symptoms and laboratory data confirmed the diagnosis.

Treatment of CMS in patients living in the Peruvian Andes may be impeded by several obstacles. Currently, relocating to lower altitudes or periodic therapeutic phlebotomy are the mainstays of therapy. These interventions pose a financial burden for the majority of patients and may contribute to poor treatment adherence. However, recent research suggests that the medication, acetazolamide, which is used primarily for the prevention of acute mountain sickness, can be helpful in cases of CMS.

The diagnosis and treatment of CMS is challenging in the remote Peruvian Andes. Education of physicians and other medical providers working in high-altitude regions requires a high index of suspicion for appropriate diagnosis and care.

Robert J. Ochsner, MD Nicholas E. Seeliger, MD

Richard L. Sundermeyer, MD

Kent T. Broberg, MD

Brandon C. Hemphill, MD John Conners, RN

Eglin Air Force Base, FL, USA

\section{Are Mountain Athletes Adequately Educated in the Evaluation and Treatment of Acute Wilderness Medicine Conditions?}

Mountain athletes are often accompanied by guides that have appropriate medical knowledge and training. Very little is known about the medical knowledge of the unguided mountain athlete. The purpose of this study was to assess the medical knowledge of unguided rock climbers, skiers, and mountaineers.

This was a prospective, cross-sectional study that surveyed adult subscribers to 2 popular rock climbing and mountaineering online magazines. The survey consisted of demographic questions, previous medical training, and an 8-question examination adapted from Paul Auerbach's Wilderness Medicine textbook and the Advanced Wilderness Life Support certification course. Descriptive statistics were calculated and differences between subgroups were compared using the MannWhitney $U$ test.

There were 111 responders to the online survey with 107 retained for analysis (average age 32.37; 96.3\% male). Most subjects had completed a first responder course $(66.4 \%)$, and half had completed a wilderness medicine course $(50.5 \%)$. Only $21.5 \%$ worked in the health care field. Overall, the group scored $72.31 \%$ on the examination. Performance varied by specific topic area as follows: trauma management (60.7\% gave the correct answer), high-altitude medicine (89.7\%), hypothermia $(99.1 \%)$, frostbite $(43.9 \%)$, avalanche $(93.5 \%)$, wound management $(67.3 \%)$, and infectious disease $(55.7 \%)$. Exam performance was better for those who had taken a wilderness medicine course $(76.85 \%$ vs $67.31 \% ; P=.002)$ or a firstresponder course $(75.35 \%$ vs $66.32 \% ; P=.004)$, or who had worked in health care $(79.89 \%$ vs $70.24 \% ; P=.01)$.

Unguided wilderness athletes likely have adequate knowledge of medical management for hypothermia, high-altitude emergencies, and avalanche rescue, but more than 1 in 3 gave the incorrect answer for management of trauma, frostbite, and infectious diseases. Previous medical training was associated with significantly higher wilderness medicine knowledge. These results may guide the development of courses to educate unguided wilderness athletes as these activities become increasingly popular.

Matthew B. Campbell, MD Jack Ditty, MD

Stephen M. Davis, MPA, MSW Morgantown, WV, USA

\section{Feasibility of the 6-Minute Walk Test in Healthy Adults at High Altitude on Aconcagua}

Background.-The 6-minute walk test (6MWT) has been used as an inexpensive, noninvasive single measurement of functional status in patients with cardiovascular diseases such as chronic obstructive pulmonary disease and congestive heart failure. The 6MWT has not been applied to the high-altitude setting.

Objective.-Perform a feasibility study of the 6MWT in a high-altitude setting.

Methods.-Study design: prospective observational cohort study. Setting: Plaza de Mulas base camp (4365 m), Aconcagua Provincial Park, Argentina. Inclusion criteria: volunteer trekkers to Aconcagua (6962 m) during January 2009 who signed written informed consent. Exclusion criteria: pregnant women, age $<18$ years. Study protocol: Subjects completed a questionnaire that included demographic information. Heart rate (HR), respiratory rate (RR), blood pressure, and oxygen saturation $\left(\mathrm{SaO}_{2}\right)$ were measured for all subjects before and after completion of a 6MWT using a standardized protocol on a 40-m course. Six-minute walk distance (6MWD) and maximal heart rate (max HR) during 6MWT were recorded. Statistical analysis: means, standard deviations, 95\% confidence intervals (CIs), and $t$ tests were calculated for normally distributed data using Stata (Stata Corp, College Station, TX).

Results.-A total of 124 subjects completed the 6MWT. One hundred nine (88\%) were male and 15 (12\%) were female. Subject demographics (mean \pm SD) were as follows: age 40.1 \pm 11.7 years; weight $75.5 \pm 10.4 \mathrm{~kg}$; height $1.77 \pm 0.10 \mathrm{~m}$; body mass index $24.2 \pm 2.8$. Mean 6MWD was $457.5 \mathrm{~m}(95 \%$ CI: 442.8-472.2; range 190-750) with max HR $115.3 \pm 13.0$ bpm. There was a significant change in HR (82.4 vs 102.4 bpm, 